\title{
Root Systems for Levi Factors and Borel-de Siebenthal Theory
}

\author{
BERTRAM KOSTANT*
}

Dedicated to Gerry Schwarz on the occasion of his 60th birthday

\begin{abstract}
Let $\mathfrak{m}$ be a Levi factor of a proper parabolic subalgebra $\mathfrak{q}$ of a complex semisimple Lie algebra $\mathfrak{g}$. Let $\mathfrak{t}=$ cent $\mathfrak{m}$. A nonzero element $\nu \in \mathfrak{t}^{*}$ is called a $\mathfrak{t}$-root if the corresponding adjoint weight space $\mathfrak{g}_{\nu}$ is not zero. If $\nu$ is a $\mathfrak{t}$-root, some time ago we proved that $\mathfrak{g}_{\nu}$ is ad $\mathfrak{m}$ irreducible. Based on this result we develop in the present paper a theory of $\mathfrak{t}$-roots which replicates much of the structure of classical root theory (case where $\mathfrak{t}$ is a Cartan subalgebra). The results are applied to obtain new results about the structure of the nilradical $\mathfrak{n}$ of $\mathfrak{q}$. Also applications in the case where $\operatorname{dim} \mathfrak{t}=1$ are used in Borel-de Siebenthal theory to determine irreducibility theorems for certain equal rank subalgebras of $\mathfrak{g}$. In fact the irreducibility results readily yield a proof of the main assertions of the Borel-de Siebenthal theory.
\end{abstract}

\section{Introduction}

0.1. Let $\mathfrak{m}$ be a Levi factor of a proper parabolic subalgebra $\mathfrak{q}$ of a complex semisimple Lie algebra $\mathfrak{g}$. Let $\mathfrak{t}=$ cent $\mathfrak{m}$ and let $\mathfrak{s}=[\mathfrak{m}, \mathfrak{m}]$ so that one has a direct $\operatorname{sum} \mathfrak{m}=\mathfrak{t}+\mathfrak{s}$. Let $\mathfrak{r}$ be the Killing form orthocomplement of $\mathfrak{m}$ in $\mathfrak{g}$ so that $\mathfrak{g}=\mathfrak{m}+\mathfrak{r}$ and $[\mathfrak{m}, \mathfrak{r}] \subset \mathfrak{r}$. A nonzero element $\nu \in \mathfrak{t}^{*}$ is called a $\mathfrak{t}$-root if $\mathfrak{g}_{\nu} \neq 0$ where $\mathfrak{g}_{\nu}=\{z \in$ $\mathfrak{g} \mid \operatorname{ad} x(z)=\nu(x) z, \forall x \in \mathfrak{t}\}$. One readily has $\mathfrak{g}_{\nu} \subset \mathfrak{r}$ and a direct sum

$$
\mathfrak{r}=\sum_{\nu \in R} \mathfrak{g}_{\nu}
$$

where $R \subset \mathfrak{t}^{*}$ is the set of all $\mathfrak{t}$-roots. It is immediate that $\mathfrak{g}_{\nu}$ is an ad $\mathfrak{m}$-submodule of $\mathfrak{r}$ for any $\nu \in R$. Some time ago we proved

Theorem 0.1. $\mathfrak{g}_{\nu}$ is an irreducible ad $\mathfrak{m}$-module for any $\nu \in R$ and any irreducible $\mathfrak{m}$-submodule of $\mathfrak{r}$ is of this form. In particular $\mathfrak{r}$ is a multiplicity-free ad $\mathfrak{m}$-module and (0.1) is the unique decomposition of $\mathfrak{r}$ as a sum of irreducible ad $\mathfrak{m}$-modules.

\footnotetext{
* Research supported in part by the KG\&G Foundation
} 
Our Theorem 0.1 appeared, with the apppropriate citations, as Theorem 8.13.3 in [Wol] and Theorem 2.1 in [Jos]. In the present paper we will use Theorem 0.1 (reproved for convenience) to develop a theory of $\mathfrak{t}$-roots which in many ways replicates results in the usual root theory, i.e., the case where $\mathfrak{t}$ is a Cartan subalgebra of $\mathfrak{g}$. For example it is established that if $\mu, \nu \in R$ and $\mu+\nu \in R$, then one has the equality

$$
\left[\mathfrak{g}_{\mu}, \mathfrak{g}_{\nu}\right]=\mathfrak{g}_{\mu+\nu}
$$

Also with respect to a natural inner product on $\mathfrak{t}^{*}$ if $\mu, \nu \in R$ and $(\mu, \nu)<0$, then $\mu+\nu \in R$, and if $(\mu, \nu)>0$, then $\mu-\nu \in R$. (See Theorem 2.2.)

The nilradical $\mathfrak{n}$ of $\mathfrak{q}$ is contained in $\mathfrak{r}$, and one introduces a set $R^{+}$of positive t-roots so that

$$
\mathfrak{n}=\sum_{\nu \in R^{+}} \mathfrak{g}_{\nu}
$$

As in the Cartan subalgebra case one can similarly define the set $R_{\text {simp }} \subset R^{+}$of simple positive $\mathfrak{t}$-roots and prove that if, by definition, $\ell(\mathfrak{t})=\operatorname{dim} \mathfrak{t}$, then $\operatorname{card} R_{\text {simp }}=\ell(\mathfrak{t})$. (See Theorem 2.7.) In fact if $R_{\text {simp }}=\left\{\beta_{1}, \ldots, \beta_{\ell(\mathfrak{t})}\right\}$, then the $\beta_{i}$ are a basis of $\mathfrak{t}^{*}$ and $\left(\beta_{i}, \beta_{j}\right) \leq 0$ if $i \neq j$. In addition one proves that $\mathfrak{n}$ is generated by $\mathfrak{g}_{\beta_{i}}$ for $i=1, \ldots, \ell(\mathfrak{t})$. In fact we prove that for the nilradical $\mathfrak{n}$ of a parabolic subalgebra of $\mathfrak{g}$ one has

Theorem 0.2. Except for indexing, the upper central series of $\mathfrak{n}$ is the same as the lower central series of $\mathfrak{n}$.

In Section 3 of the paper we deal with the case where $\ell(\mathfrak{t})=1$ so that $\mathfrak{q}$ is a maximal parabolic subalgebra. The results are applied here to Borel-de Siebenthal theory and irreducibility theorems are obtained for the adjoint action of equal (to that of $\mathfrak{g}$ ) rank subalgebras $\mathfrak{g}^{a_{j}}$ of $\mathfrak{g}$ on the Killing form orthocomplement of $\mathfrak{g}^{a_{j}}$ in $\mathfrak{g}$. In Remark 3.9 we also show that these results provide a proof of the main statements of the Borel-de Siebenthal theory.

\section{Levi factor root system}

1.1. Let $\mathfrak{g}$ be a complex semisimple Lie algebra. Let $\mathfrak{h}$ be a Cartan subalgebra of $\mathfrak{g}$ and let $\ell$ be the rank of $\mathfrak{g}$. Let $\mathfrak{h}^{*}$ be the dual space to $\mathfrak{h}$ and let $\Delta \subset \mathfrak{h}^{*}$ be 
the set of roots for the pair $(\mathfrak{h}, \mathfrak{g})$. For each $\varphi \in \Delta$, let $e_{\varphi} \in \mathfrak{g}$ be a corresponding root vector. Let $\Delta_{+} \subset \Delta$ be a choice of positive roots and let $\Pi \subset \Delta$ be the set of simple positive roots. Let $\Delta_{-}=-\Delta_{+}$. For any $\varphi \in \Delta$ and $\alpha \in \Pi$, let $n_{\alpha}(\varphi) \in \mathbb{Z}$ be the integer (nonnegative if $\varphi \in \Delta_{+}$and nonpositive if $\varphi \in \Delta_{-}$) so that

$$
\varphi=\sum_{\alpha \in \Pi} n_{\alpha}(\varphi) \alpha
$$

If $\mathfrak{u} \subset \mathfrak{g}$ is any subspace which is stable under ad $\mathfrak{h}$, let

$$
\begin{aligned}
\Delta(\mathfrak{u}) & =\left\{\varphi \in \Delta \mid e_{\varphi} \in \mathfrak{u}\right\} \\
\Delta_{+}(\mathfrak{u}) & =\Delta(\mathfrak{u}) \cap \Delta_{+} \\
\Delta_{-}(\mathfrak{u}) & =\Delta(\mathfrak{u}) \cap \Delta_{-} .
\end{aligned}
$$

Let $\mathfrak{b}$ be the Borel subalgebra of $\mathfrak{g}$, containing $\mathfrak{h}$ such that $\Delta(\mathfrak{b})=\Delta_{+}$. Let $\mathfrak{n}_{\mathfrak{b}}=[\mathfrak{b}, \mathfrak{b}]$ be the nilradical of $\mathfrak{b}$. A standard parabolic subalgebra $\mathfrak{q}$ of $\mathfrak{g}$ is any Lie subalgebra of $\mathfrak{g}$ which contains $\mathfrak{b}$.

Let $B$ be the Killing form on $\mathfrak{g}$. Assume that $\mathfrak{q}$ is some fixed standard parabolic subalgebra of $\mathfrak{g}$. Then $\mathfrak{q}$ admits a unique Levi decomposition

$$
\mathfrak{q}=\mathfrak{m}+\mathfrak{n}
$$

where $\mathfrak{n}=\mathfrak{n}_{\mathfrak{q}}\left(\subset \mathfrak{n}_{\mathfrak{b}}\right)$ is the nilradical of $\mathfrak{q}$ and $\mathfrak{m}=\mathfrak{m}_{\mathfrak{q}}$ is the unique Levi factor of $\mathfrak{q}$ which contains $\mathfrak{h}$. We will assume throughout that $\mathfrak{q} \neq \mathfrak{g}$ so that $\mathfrak{n} \neq 0$. Let $\mathfrak{s}=[\mathfrak{m}, \mathfrak{m}]$ so that $\mathfrak{s}$ is the unique maximal semisimple ideal in $\mathfrak{m}$. Let $\mathfrak{t}$ be the center of $\mathfrak{m}$ so that $B$ is nonsingular on both $\mathfrak{t}$ and $\mathfrak{m}$ and

$$
\mathfrak{m}=\mathfrak{t} \oplus \mathfrak{s}
$$

is a $B$-orthogonal decomposition of $\mathfrak{m}$ into a direct sum of ideals. Let $\mathfrak{h}(\mathfrak{s})=\mathfrak{h} \cap \mathfrak{s}$ so that $\mathfrak{h}(\mathfrak{s})$ is a Cartan subalgebra of $\mathfrak{s} . B$ is nonsingular on $\mathfrak{h}(\mathfrak{s})$ and

$$
\mathfrak{h}=\mathfrak{t} \oplus \mathfrak{h}(\mathfrak{s})
$$

is a $B$-orthogonal decomposition.

The nonsingular bilinear form, $B \mid \mathfrak{h}$, on $\mathfrak{h}$ induces a nonsingular bilinear form on $\mathfrak{h}^{*}$ which we denote by $B \mid \mathfrak{h}^{*}$. We may embed the dual spaces $\mathfrak{t}^{*}$ and $\mathfrak{h}(\mathfrak{s})^{*}$ to $\mathfrak{t}$ and 
$\mathfrak{h}(\mathfrak{s})$, respectively, in $\mathfrak{h}^{*}$ so that $\mathfrak{t}^{*}$ is the orthocomplement of $\mathfrak{h}(\mathfrak{s})$ and $\mathfrak{h}(\mathfrak{s})^{*}$ is the orthocomplement to $\mathfrak{t}$. Then $B \mid \mathfrak{h}^{*}$ is nonsingular on both $\mathfrak{t}^{*}$ and $\mathfrak{h}(\mathfrak{s})^{*}$ and

$$
\mathfrak{h}^{*}=\mathfrak{t}^{*} \oplus \mathfrak{h}(\mathfrak{s})^{*}
$$

is a $B \mid \mathfrak{h}^{*}$ orthogonal direct sum.

Let $\mathfrak{h}_{\mathbb{R}}^{*}$ be the real form of $\mathfrak{h}^{*}$ spanned over $\mathbb{R}$ by $\Delta$. As one knows, $B \mid \mathfrak{h}^{*}$ is positive definite on $\mathfrak{h}_{\mathbb{R}}^{*}$. On the other hand, similarly, $\Delta(\mathfrak{s})$ clearly spans over $\mathbb{R}$, a real form, $\mathfrak{h}(\mathfrak{s})_{\mathbb{R}}^{*}$, of $\mathfrak{h}(\mathfrak{s})^{*}$. Of course $\mathfrak{h}(\mathfrak{s})_{\mathbb{R}}^{*}$ is a real subspace of $\mathfrak{h}_{\mathbb{R}}^{*}$ and, clearly, if $\mathfrak{t}_{\mathbb{R}}^{*}$ is the $B \mid \mathfrak{h}$ * orthocomplement of $\mathfrak{h}(\mathfrak{s})_{\mathbb{R}}^{*}$ in $\mathfrak{h}_{\mathbb{R}}^{*}$, then $\mathfrak{t}_{\mathbb{R}}^{*}$ is a real form of $\mathfrak{t}^{*}$ and

$$
\mathfrak{h}_{\mathbb{R}}^{*}=\mathfrak{t}_{\mathbb{R}}^{*} \oplus \mathfrak{h}(\mathfrak{s})_{\mathbb{R}}^{*}
$$

is a real Hilbert space orthogonal direct sum. For any $\gamma \in \mathfrak{h}_{\mathbb{R}}^{*}$ we let $\gamma_{\mathfrak{t}} \in \mathfrak{t}_{\mathbb{R}}^{*}$ and $\gamma_{\mathfrak{s}} \in \mathfrak{h}(\mathfrak{s})_{\mathbb{R}}^{*}$, respectively, be the components of $\gamma$ with respect to the decomposition (1.7) so that

$$
\gamma=\gamma_{\mathfrak{t}}+\gamma_{\mathfrak{s}}
$$

and

$$
\left(\gamma_{\mathfrak{t}}, \gamma_{\mathfrak{s}}\right)=0
$$

where $(\mu, \lambda)$ denotes the $B \mid \mathfrak{h}^{*}$-pairing of any $\mu, \lambda \in \mathfrak{h}^{*}$.

Let $\overline{\mathfrak{n}}$ be the span of all $e_{-\varphi}$, for $\varphi \in \Delta(\mathfrak{n})$ so that one has a triangular decomposition

$$
\mathfrak{g}=\mathfrak{m}+\mathfrak{n}+\overline{\mathfrak{n}}
$$

Now put $\mathfrak{r}=\mathfrak{n}+\overline{\mathfrak{n}}$ so that $\mathfrak{r}$ is ad $\mathfrak{m}$-stable and one has a $B$-orthogonal decomposition

$$
\mathfrak{g}=\mathfrak{m}+\mathfrak{r}
$$

Remark 1.1. From the general properties of Levi factors of parabolic subalgebras one knows that $\mathfrak{m}$ is the centralizer of $\mathfrak{t}$ in $\mathfrak{g}$ so that

$$
\Delta(\mathfrak{r})=\left\{\varphi \in \Delta \mid \varphi_{\mathfrak{t}} \neq 0\right\}
$$

1.2. Let $V$ be a $\mathfrak{t}$-module and let $\mu \in \mathfrak{t}^{*}$. Put

$$
V_{\mu}=\{v \in V \mid x \cdot v=\langle\mu, x\rangle v, \forall x \in \mathfrak{t}\} .
$$


The subspace $V_{\mu}$ is called the $\mu$-weight space (for $\mathfrak{t}$ ) of $V$. If $V_{\mu} \neq 0$, then $\mu$ is called a t-weight of $V$ and any $v \in V_{\mu}$ is called a $\mu$-weight vector.

If $V$ is a finite-dimensional $\mathfrak{g}$-module, then necessarily $\gamma \in \mathfrak{h}_{\mathbb{R}}^{*}$ where $\gamma$ is any $\mathfrak{h}$-weight of $V$. One then notes $\mu \in \mathfrak{t}^{*}$ is a $\mathfrak{t}$-weight of $V$ if and only if

$$
\mu=\gamma_{\mathfrak{t}} \text { where } \gamma \text { is an } \mathfrak{h} \text {-weight of } V \text {. }
$$

An important special case is when $V=\mathfrak{g}$ and the module structure is defined by the adjoint action. Let $R^{\prime}$ be the set of all $\mathfrak{t}$-weights of $\mathfrak{g}$. If $V$ is any $\mathfrak{g}$-module and $\xi$ is a $\mathfrak{t}$-weight of $V$, it is obvious that, for any $\mu \in R^{\prime}$,

$$
\mathfrak{g}_{\mu} \cdot V_{\xi} \subset V_{\mu+\xi}
$$

Clearly $0 \in R^{\prime}$ and

$$
\mathfrak{g}_{0}=\mathfrak{m}
$$

so that $V_{\xi}$ is an $\mathfrak{m}$-module.

Remark 1.2. If $V$ is finite dimensional then, since $\mathfrak{s} \subset \mathfrak{m}$ and $\mathfrak{s}$ is semisimple, note that $V_{\xi}$ is a completely reducible $\mathfrak{s}$-module.

If $V$ is equal to the adjoint $\mathfrak{g}$-module $\mathfrak{g}$ in $(1.15)$ one has

$$
\left[\mathfrak{g}_{\nu}, \mathfrak{g}_{\mu}\right] \subset \mathfrak{g}_{\nu+\mu}
$$

for any $\nu, \mu \in R^{\prime}$. In particular, if $\mu \in R^{\prime}$, then

$$
\left[\mathfrak{m}, \mathfrak{g}_{\mu}\right] \subset \mathfrak{g}_{\mu}
$$

and

$\mathfrak{g}_{\mu}$ is a completely reducible $\mathfrak{s}$-module for the maximal semisimple ideal $\mathfrak{s}$ of $\mathfrak{m}$.

Let $R=R^{\prime} \backslash\{0\}$ so that, recalling Remark 1.1,

$$
R=\left\{\nu \in \mathfrak{t}^{*} \mid \nu=\varphi_{\mathfrak{t}}, \text { for some } \varphi \in \Delta(\mathfrak{r})\right\},
$$

and one readily has (see (1.16)) the direct sum

$$
\mathfrak{g}=\mathfrak{m}+\sum_{\nu \in R} \mathfrak{g}_{\nu}
$$


We refer to the elements in $R$ as $\mathfrak{t}$-roots (in $\mathfrak{g}$ ) and $\mathfrak{g}_{\nu}$ as the $\mathfrak{t}$-root space corresponding to $\nu \in R$. Partially summarizing one readily has

Proposition 1.3. One has the two disjoint unions

$$
\begin{aligned}
\Delta & =\sqcup_{\mu \in R^{\prime}} \Delta\left(\mathfrak{g}_{\mu}\right) \\
\Delta(\mathfrak{r}) & =\sqcup_{\nu \in R} \Delta\left(\mathfrak{g}_{\nu}\right) .
\end{aligned}
$$

Furthermore if $\nu \in R$, then

$$
\begin{gathered}
\Delta\left(\mathfrak{g}_{\nu}\right)=\left\{\varphi \in \Delta \mid \varphi_{\mathfrak{t}}=\nu\right\} \text { and } \\
\left\{e_{\varphi} \mid \varphi \in \Delta\left(\mathfrak{g}_{\nu}\right), \text { is a basis of } \mathfrak{g}_{\nu}\right\} .
\end{gathered}
$$

Let $(x, y)$ denote the pairing of $x, y \in \mathfrak{g}$ defined by $B$.

Remark 1.4. Note that $\nu \in R$ if and only if $-\nu \in R$ and

$$
\Delta\left(\mathfrak{g}_{-\nu}\right)=-\Delta\left(\mathfrak{g}_{\nu}\right)
$$

Furthermore if $\mu, \nu \in R$ and $\nu \neq-\mu$, then

$$
\left(\mathfrak{g}_{\mu}, \mathfrak{g}_{\nu}\right)=0
$$

and

$$
\mathfrak{g}_{\nu} \text { and } \mathfrak{g}_{-\nu} \text { are nonsingularly paired by } B \text {. }
$$

1.3. Let $\tau: \mathfrak{h} \rightarrow \mathfrak{h}^{*}$ be the linear isomorphism defined by $B \mid \mathfrak{h}$. Thus for $x \in \mathfrak{h}$ and $\mu \in \mathfrak{h}^{*}$,

$$
\begin{aligned}
\langle\mu, x\rangle & =(\mu, \tau(x)) \\
& =\left(\tau^{-1}(\mu), x\right) .
\end{aligned}
$$

Thus if $\mathfrak{t}_{\mathbb{R}}=\tau^{-1}\left(\mathfrak{t}_{\mathbb{R}}^{*}\right)$, then one readily has

\section{Proposition 1.5.}

(1) $\mathfrak{t}_{\mathbb{R}}$ is a real form of $\mathfrak{t}$

(2) $B$ is real and positive definite on $\mathfrak{h}_{\mathbb{R}}$

(3) $\mathfrak{t}_{\mathbb{R}}=\{x \in \mathfrak{t} \mid\langle\nu, x\rangle$ is real $\forall \nu \in R\}$. 
Let $\nu \in R$. Clearly $\operatorname{Ker} \nu$ has codimension 1 in $\mathfrak{t}$. It follows from Proposition 1.5 that there exists a unique element $h_{\nu} \in \mathfrak{t}_{\mathbb{R}}$ which is $B$-orthogonal to Ker $\nu$ and such that

$$
\left\langle\nu, h_{\nu}\right\rangle=2
$$

Note that it follows from (1.27) that

$$
\tau\left(h_{\tau}\right)=2 \nu /(\nu, \nu)
$$

Let $\nu \in R$ and put $\mathfrak{m}(\nu)=\left[\mathfrak{g}_{\nu}, \mathfrak{g}_{-\nu}\right]$ so that $\mathfrak{m}(\nu)$ is an ideal of $\mathfrak{m}$. Let $\mathfrak{t}(\nu)=$ $\mathfrak{m}(\nu) \cap \mathfrak{t}$ and $\mathfrak{s}(\nu)=\mathfrak{m}(\nu) \cap \mathfrak{s}$. Decomposing the adjoint action representation of $\mathfrak{s}$ on $\mathfrak{m}(\nu)$ into its primary components, it is clear that $\mathfrak{t}(\nu)$ is the primary component corresponding to the trivial representation and $\mathfrak{s}(\nu)$ is the sum of the remaining components. Consequently one has the direct sum

$$
\mathfrak{m}(\nu)=\mathfrak{t}(\nu) \oplus \mathfrak{s}(\nu)
$$

Remark 1.6. If $\mathfrak{s}_{i}$ is a simple component of $\mathfrak{s}$, one has $\Delta\left(\mathfrak{s}_{i}\right)=-\Delta\left(\mathfrak{s}_{i}\right)$ so that $B \mid \mathfrak{s}_{i}$ is nonzero and hence nonsingular by simplicity. On the other hand if $\mathfrak{s}_{i}$ and $\mathfrak{s}_{j}$ are distinct simple components then, of course, $\left[\mathfrak{s}_{i}, \mathfrak{s}_{j}\right]=0$. Consequently $\mathfrak{s}_{i}$ and $\mathfrak{s}_{j}$ are $B$-orthogonal by the invariance of $B$ and the equality $\left[\mathfrak{s}_{i}, \mathfrak{s}_{i}\right]=\mathfrak{s}_{i}$.

Proposition 1.7. Let $\nu \in R$. Then

$$
\mathfrak{t}(\nu)=\mathbb{C} h_{\nu}
$$

In addition $B \mid \mathfrak{m}(\nu)$ is nonsingular and the kernel of the adjoint action of $\mathfrak{m}$ on $\mathfrak{g}_{\nu}$ is the orthocomplement of $\mathfrak{m}(\nu)$ in $\mathfrak{m}$. In particular $\mathfrak{m}(\nu)$ operates faithfully on $\mathfrak{g}_{\nu}$.

Proof. Let $\mathfrak{m}(\nu)^{\perp}$ be the $B$-orthogonal subspace to $\mathfrak{m}(\nu)$ in $\mathfrak{m}$. Let $x \in \mathfrak{m}, y \in \mathfrak{g}_{\nu}$, and $z \in \mathfrak{g}_{-\nu}$. But $(x,[y, z])=([x, y], z)$. Since $\mathfrak{g}_{\nu}$ and $\mathfrak{g}_{-\nu}$ are orthogonally paired by $B$ this proves that $\mathfrak{m}(\nu)^{\perp}$ is the kernel of the adjoint action of $\mathfrak{m}$ on $\mathfrak{g}_{\nu}$. But then $\operatorname{Ker} \nu=\mathfrak{m}(\nu)^{\perp} \cap \mathfrak{t}$. Recalling the definition and properties of $h_{\nu}$ (see (1.29) and Proposition 1.5) it follows immediately that $B \mid \operatorname{Ker} \nu$ is nonsingular. It then follows that $\mathfrak{t}(\nu)$ must be the one-dimensional $B$-orthocomplement of $\operatorname{Ker} \nu$ in $\mathfrak{t}$. But then one has (1.32) by definition of $h_{\nu}$. But now $\mathfrak{s}(\nu)$ is clearly an ideal in $\mathfrak{s}$. Hence $\mathfrak{s}(\nu)$ is a sum of simple components of $\mathfrak{s}$. Thus $B \mid \mathfrak{m}(\nu)$ is nonsingular by Remark 1.6. QED 
1.3. In this section we will mainly be concerned with decomposing $\mathfrak{r}$ into irreducible $\mathfrak{m}$-modules. Effectively this comes down to understanding the action of $\mathfrak{s}$ on $\mathfrak{g}_{\nu}$ for any $\nu \in R$.

Obviously $\Delta_{+}(\mathfrak{s})$ defines a choice of positive roots in $\Delta(\mathfrak{s})$ so that

$$
\mathfrak{b}(\mathfrak{s})=\mathfrak{h}(\mathfrak{s})+\sum_{\varphi \in \Delta_{+}(\mathfrak{s})} \mathbb{C} e_{\varphi}
$$

is a Borel subalgebra of $\mathfrak{s}$. Highest weights and highest weight vectors for $\mathfrak{s}$-modules will be defined with respect to $\mathfrak{b}(\mathfrak{s})$.

Let $C(\mathfrak{s}) \subset \mathfrak{h}(\mathfrak{s})_{\mathbb{R}}^{*}$ be the dominant Weyl chamber.

Proposition 1.8. Let $\xi, \eta \in C(\mathfrak{s})$. Then

$$
(\xi, \eta) \geq 0 .
$$

Proof. Let $I$ be an index set for the simple components of $\mathfrak{s}$ where, if $i \in I$, then $\mathfrak{s}_{i}$ is the corresponding component. One readily has

$$
C(\mathfrak{s})=\sum_{i \in I} C\left(\mathfrak{s}_{i}\right)
$$

where $\mathfrak{h}\left(\mathfrak{s}_{i}\right)=\mathfrak{h} \cap \mathfrak{s}_{i}$ and $C\left(\mathfrak{s}_{i}\right) \subset \tau\left(\mathfrak{h}\left(\mathfrak{s}_{i}\right)\right)$ is the dominant Weyl chamber for $\mathfrak{s}_{i}$. But then, if $i, j \in I$ are distinct, $C\left(\mathfrak{s}_{i}\right)$ and $C\left(\mathfrak{s}_{j}\right)$ are $B \mid \mathfrak{h}^{*}$-orthogonal by (1.27) and Remark 1.6. Thus it suffices to prove that, if $i \in I$, and $\xi, \eta \in C\left(\mathfrak{s}_{i}\right)$ are nonzero, then

$$
(\xi, \eta)>0 .
$$

But $B \mid \mathfrak{s}_{i}$ is a positive multiple of the $\mathfrak{s}_{i}$-Killing form and for the $\mathfrak{s}_{i}$-Killing form (1.34) is known (see e.g., Lemma 2.4 in $[\mathrm{Kos}]$ ). QED

We established the following theorem some time ago. It appears in the literature in works of J. Wolf and A. Joseph with proper citations in both cases. See Theorem 8.13.3 in [Wol] and in a closer reproduction of my argument, Theorem 2.1 in [Jos].

Theorem 1.9. Let $\nu \in R$. Then the $\mathfrak{t}$-root subspace $\mathfrak{g}_{\nu}$ is an irreducible $\mathfrak{m}$ and irreducible $\mathfrak{s - m o d u l e ~ u n d e r ~ t h e ~ a d j o i n t ~ a c t i o n . ~ I n ~ f a c t ~ i t ~ i s ~ a ~ f a i t h f u l ~ i r r e d u c i b l e ~}$ $\left[\mathfrak{g}_{\nu}, \mathfrak{g}_{-\nu}\right] \subset \mathfrak{m}$-module and in the notation of (1.31) an irreducible $\mathfrak{s}(\nu)$-module. In addition

$$
\mathfrak{r}=\sum_{\nu \in R} \mathfrak{g}_{\nu}
$$


is a multiplicity-one representation of $\mathfrak{m}$ and the summands (i.e., the $\mathfrak{t}$-root spaces) on the right hand side of (1.35) are the irreducible components.

Proof. Each of the summands on the right side of (1.35) affords a different character of $\mathfrak{t}$ and hence these summands are inequivalent as $\mathfrak{m}$-modules. Recalling Proposition 1.7 it suffices only to prove that $\mathfrak{g}_{\nu}$ is an irreducible $\mathfrak{s}$-module. The elements $\left\{e_{\varphi} \mid \varphi \in \Delta\left(\mathfrak{g}_{\nu}\right)\right\}$ are a weight basis of $\mathfrak{g}_{\nu}$ for the Cartan subalgebra $\mathfrak{h}(\mathfrak{s})$. Moreover, since root spaces for $\mathfrak{h}$ have multiplicity-one, the $\mathfrak{h}(\mathfrak{s})$-weights in $\mathfrak{g}_{\nu}$ have multiplicity-one since $\varphi_{\mathfrak{t}}=\varphi_{\mathfrak{t}}^{\prime}$ for $\varphi, \varphi^{\prime} \in \Delta\left(\mathfrak{g}_{\nu}\right)$.

Assume $\mathfrak{g}_{\nu}$ is not $\mathfrak{s}$-irreducible. Then there exists distinct $\varphi, \varphi^{\prime} \in \Delta\left(\mathfrak{g}_{\nu}\right)$ such that $e_{\varphi}$ and $e_{\varphi^{\prime}}$ are $\mathfrak{s}$-highest weight vectors. In particular $\varphi_{\mathfrak{s}}$ and $\varphi_{\mathfrak{s}}^{\prime}$ are in $C(\mathfrak{s})$. But then

$$
\left(\varphi_{\mathfrak{s}}, \varphi_{\mathfrak{s}}^{\prime}\right) \geq 0
$$

by Proposion 1.8. But

$$
\begin{aligned}
\varphi & =\nu+\varphi_{\mathfrak{s}} \\
\varphi^{\prime} & =\nu+\varphi_{\mathfrak{s}}^{\prime} .
\end{aligned}
$$

Hence

$$
\left(\varphi, \varphi^{\prime}\right)>0
$$

Thus $\beta=\varphi-\varphi^{\prime}$ is a root. Furthermore $\beta_{\mathfrak{t}}=0$ so that $\beta \in \Delta(\mathfrak{m})=\Delta(\mathfrak{s})$. Without loss of generality we may choose the ordering so that $\beta \in \Delta_{+}(\mathfrak{s})$. But then $\left[e_{\beta}, e_{\varphi^{\prime}}\right]$ is a nonzero multiple of $e_{\varphi}$. This contradicts the fact that $e_{\varphi^{\prime}}$ is an $\mathfrak{s}$-highest weight vector. QED

\section{Properties of the $t$-root system}

2.1. We will utilize Theorem 1.9 to establish some properties of $R$. To begin with

Lemma 2.1. Assume $\nu, \mu \in R$ and $\nu+\mu \neq 0$. Assume also that $\left[\mathfrak{g}_{\nu}, \mathfrak{g}_{\mu}\right] \neq 0$. Then $\nu+\mu \in R$ (obvious) and one has the equality

$$
\left[\mathfrak{g}_{\nu}, \mathfrak{g}_{\mu}\right]=\mathfrak{g}_{\nu+\mu}
$$


Proof. The left side of $(2.1)$ is a nonzero $\mathfrak{m}$-submodule of the right side. One therefore has the equality (2.1) by irreducibility. QED

Let $p, q \in \mathbb{Z}$ where $p \leq q$. Let $I_{p, q}$ denote the set of integers $m$ such that $p \leq m \leq q$. A finite nonempty subset $I \subset \mathbb{Z}$ will be called an interval if it is of the form $I_{p, q}$.

Theorem 2.2. Let $\nu \in R$ and assume $V$ is a finite-dimensional $\mathfrak{g}$-module with respect to a representation $\pi$. Let $\gamma$ be a $\mathfrak{t}$-weight of $V$ and let

$$
I=\{j \in \mathbb{Z} \mid \gamma+j \nu \text { be a } \mathfrak{t} \text {-weight of } V\}
$$

noting that $I$ is of course finite and not empty since $0 \in I$. Then there exist $p \leq 0 \leq$ $q, p, q \in \mathbb{Z}$ such that

$$
I=I_{p, q}
$$

Moreover if I has only one element (i.e., $p=q=0)$, then $(\gamma, \nu)=0$. Furthermore if I has more than one element (i.e., $p<q$ ), then

$$
\begin{aligned}
& (\gamma+q \nu, \nu)>0 \text { and } \\
& (\gamma+p \nu, \nu)<0
\end{aligned}
$$

Finally let $m \in I_{p, q}$. If $m<q$. Then

$$
\pi\left(\mathfrak{g}_{\nu}\right)\left(V_{\gamma+m \nu}\right) \neq 0
$$

and if $p<m$, then

$$
\pi\left(\mathfrak{g}_{-\nu}\right)\left(V_{\gamma+m \nu}\right) \neq 0
$$

Proof. Let $X=\sum_{j \in I} V_{\gamma+j \nu}$ so that $X$ is stable under $\pi\left(\mathfrak{g}_{\nu}\right)$ and $\pi\left(\mathfrak{g}_{-\nu}\right)$ as well as $\pi(\mathfrak{m})$. One notes that, by $(1.30)$, if $j \in I$, then

$V_{\gamma+j \nu}$ is the eigenspace of $\pi\left(h_{\nu}\right) \mid X$ corresponding to the eigenvalue $\left\langle\gamma, h_{\nu}\right\rangle+2 j$.

Now since $h_{\nu} \in\left[\mathfrak{g}_{\nu}, \mathfrak{g}_{-\nu}\right]$ one must have

$$
\operatorname{tr} \pi\left(h_{\nu}\right) \mid Y=0
$$


where $Y \subset X$ is any subspace which is stable under $\pi\left(\mathfrak{g}_{\nu}\right)$ and $\pi\left(\mathfrak{g}_{-\nu}\right)$. If $I$ has one element, then obviously $I=I_{0,0}$ and one has $(\gamma, \nu)=0$ by $(2.7)$. Thus it suffices to consider the case where $I$ has more than one element. Now if $Y_{1}, Y_{2}$ are two nonzero subspaces of $X$ that are both stable under $\pi\left(\mathfrak{g}_{\nu}\right)$ and $\pi\left(\mathfrak{g}_{-\nu}\right)$, it follows from $(2.7)$ that one cannot have that the

maximal eigenvalue of $\pi\left(h_{\nu}\right)$ in $Y_{1}$ i minimal eigenvalue of $\pi\left(h_{\nu}\right)$ in $Y_{2}$.

Now assume that $p, q \in I$ and $m \in \mathbb{Z}$ is such that $m \notin I$ and $p<m<q$. If we define $Y_{1}$ (resp. $Y_{2}$ ) to be the sum of all $V_{\gamma+j}$, where $j \in I$ and $j<m$ (resp. $j>m$ ), the conditions of (2.8) are satisfied which, as noted above, is a contradiction. Thus $I=I_{p, q}$ for some $p, q \in \mathbb{Z}$ where $q>p$. But then (2.3) follows from (2.7) where $Y=X$.

Now let $m \in I_{p, q}$ where $m<q$. Assume that $\pi\left(\mathfrak{g}_{\tau}\right)\left(V_{\gamma+m \nu}\right)=0$. That is,

$$
\pi\left(e_{\varphi}\right)\left(V_{\gamma+m \nu}\right)=0, \forall \varphi \in \Delta\left(\mathfrak{g}_{\nu}\right)
$$

Thus for any $v \in V_{\gamma+(m+1) \nu}$ and $\varphi \in \Delta\left(\mathfrak{g}_{\nu}\right)$, one has

$$
\pi\left(e_{\varphi}\right) \pi\left(e_{-\varphi}\right) v=0
$$

But from the representation theory of three-dimensional simple Lie algebras, (2.10) implies that $\pi\left(e_{-\varphi}\right) v=0$. That is

$$
\pi\left(\mathfrak{g}_{-\nu}\right)\left(V_{\gamma+(m+1) \nu}\right)=0
$$

But then if $Y_{1}$ (resp. $Y_{2}$ ) is the sum of all $V_{\gamma+j \nu}$ for $j \in I_{p, q}$ where $j \leq m$ (resp. $j \geq m+1$ ), one defines $Y_{1}$ and $Y_{2}$ satisfying the contradictory (2.8). This proves (2.4). Clearly a similar argument proves (2.5). QED

Applying Theorem 2.2 and Lemma 2.1 to the case where $V=\mathfrak{g}$ and $\pi$ is the adjoint representation, one immediately has the following result asserting that some familar properties of ordinary roots still hold for $\mathfrak{t}$-roots.

Theorem 2.3. Let $\nu, \mu \in R$. If $\mu+\nu \in R$ (resp. $\mu-\nu \in R$ ), then

$$
\begin{aligned}
{\left[\mathfrak{g}_{\mu}, \mathfrak{g}_{\nu}\right] } & =\mathfrak{g}_{\nu+\mu}(\text { resp } . \\
{\left[\mathfrak{g}_{\mu}, \mathfrak{g}_{-\nu}\right] } & \left.=\mathfrak{g}_{\mu-\nu}\right) .
\end{aligned}
$$


Furthermore, one indeed has

$\mu+\nu \in R($ resp. $\mu-\nu \in R)$ if $(\mu, \nu)<0($ resp. $(\mu, \nu)>0)$ and $\mu+\nu \neq 0($ resp. $\mu-\nu \neq 0)$.

Moreover

$$
\text { if }(\mu, \nu)=0 \text { then } \mu+\nu \in R \text { if and only if } \mu-\nu \in R \text {. }
$$

2.2. Recalling (1.3) let $\delta_{\mathfrak{n}}$ be in the dual space $\mathfrak{m}^{*}$ to $\mathfrak{m}$ defined so that if $x \in \mathfrak{m}$, then

$$
\left\langle\delta_{\mathfrak{n}}, x\right\rangle=\operatorname{tr} \operatorname{ad} x \mid \mathfrak{n} .
$$

Since $\mathfrak{n}$ and $\overline{\mathfrak{n}}$ (see (1.10)) are clearly stable under ad $\mathfrak{m}$ one has a partition $R=R_{\mathfrak{n}} \cup R_{\overline{\mathfrak{n}}}$ so that

$$
\begin{aligned}
& \mathfrak{n}=\sum_{\nu \in R_{\mathfrak{n}}} \mathfrak{g}_{\nu} \\
& \overline{\mathfrak{n}}=\sum_{\nu \in R_{\overline{\mathfrak{n}}}} \mathfrak{g}_{\nu} .
\end{aligned}
$$

Clearly Remark 1.4 implies

$$
R_{\overline{\mathfrak{n}}}=-R_{\mathfrak{n}} .
$$

Lemma 2.4. One has $\delta_{\mathfrak{n}} \in \mathfrak{t}_{\mathbb{R}}^{*}($ see (1.7)). Furthermore

$$
\begin{aligned}
& \left(\delta_{\mathfrak{n}}, \nu\right)>0, \text { if } \nu \in R_{\mathfrak{n}} \text { and } \\
& \left(\delta_{\mathfrak{n}}, \nu\right)<0, \text { if } \nu \in R_{\overline{\mathfrak{n}}} .
\end{aligned}
$$

Proof. Since $\mathfrak{s}$ is semisimple, $\delta_{\mathfrak{n}}$ must vanish on $\mathfrak{s}$ and hence

$$
\delta_{\mathfrak{n}} \in \mathfrak{t}^{*}
$$

where, besides regarding $\mathfrak{t}^{*} \subset \mathfrak{h}^{*}$ as in (1.6), we also regard $\mathfrak{t}^{*} \subset \mathfrak{m}^{*}$, using (1.4). Let $\varphi \in \Delta$. Normalize the choice of root vectors so that $\left(e_{\varphi}, e_{-\varphi}\right)=1$. Let $\mathfrak{a}_{\varphi}$ be the root TDS corresponding to $\varphi$ and let $x_{\varphi}=\left[e_{\varphi}, e_{-\varphi}\right]$. Then as one knows $x_{\varphi} \in \mathfrak{h}_{\mathbb{R}}^{*}$ and (see $(1.27))$

$$
\tau\left(x_{\varphi}\right)=\varphi
$$

For some index set $P$, let

$$
\mathfrak{g}=\sum_{p \in P} \mathfrak{u}_{p}
$$


be the decomposition of $\mathfrak{g}$ into a sum of irreducible ad $\left(\mathfrak{a}_{\varphi}+\mathfrak{h}\right)$-submodules. One clearly has the direct sum (with possibly 0-dimensional summands)

$$
\mathfrak{n}=\sum_{p \in P} \mathfrak{n}_{p}
$$

where $\mathfrak{n}_{p}=\mathfrak{n} \cap \mathfrak{u}_{p}$. Now assume that $\varphi \in \Delta(\mathfrak{n})$. Then since each $\mathfrak{n}_{p}$ is stable under ad $e_{\varphi}$ for any $p \in P$, it is immediate from the representation theory of a TDS that

$$
\operatorname{tr} \operatorname{ad} x_{\varphi} \mid \mathfrak{n}_{p} \geq 0
$$

But there exists $p_{o} \in P$ such that $\mathfrak{n}_{p_{o}}=\mathbb{C} e_{\varphi}$ so that $\operatorname{tr} \operatorname{ad} x_{\varphi} \mid \mathfrak{n}_{p_{o}}>0$. Thus

$$
\delta_{\mathfrak{n}}\left(x_{\varphi}\right)>0
$$

But then

$$
\left(\delta_{\mathfrak{n}}, \varphi\right)>0
$$

by (2.20). Now let $\nu \in R_{\mathfrak{n}}$ and let $\varphi \in \Delta\left(\mathfrak{g}_{\nu}\right)$. But clearly $\nu=\varphi_{\mathfrak{t}}$ (see (1.23)) so that (2.24) and (2.19) imply the first line of (2.18). The second line is implied by (2.17). Since $\mathfrak{h}_{\mathbb{R}}^{*}$ is clearly spanned by $R_{\mathfrak{n}}$ (see $(1.28)$ ), it follows from $(2.18)$ and $(2.10)$ that $\delta_{\mathfrak{n}} \in \mathfrak{t}_{\mathbb{R}}^{*} \cdot \mathrm{QED}$

Introduce the lexicographical ordering in $\mathfrak{t}_{\mathbb{R}}^{*}$ with respect to an orthogonal ordered basis of $\mathfrak{t}_{\mathbb{R}}^{*}$ having $\delta_{\mathfrak{n}}$ as its first element. It follows from Lemma 2.4 that if $R_{+}$is the set of positive $\mathfrak{t}$-roots with respect to this ordering, one has

$$
R_{+}=R_{\mathfrak{n}}
$$

Remark 2.5. One recalls that since $\mathfrak{t}_{\mathbb{R}}^{*}$ is a lexicographically ordered real euclidean space if $\xi_{i} \in \mathfrak{t}_{\mathbb{R}}^{*}, i=1, \ldots, k$, are positive elements such that, for $i \neq j$,

$$
\left(\xi_{i}, \xi_{j}\right) \leq 0
$$

then the $\xi_{i}$ are linearly independent. ( See e.g., [Hum], §10, Theorem', (3), p. 48.)

2.3. Let $\ell(\mathfrak{t})=\operatorname{dim} \mathfrak{t}$ and $\ell(\mathfrak{s})=\operatorname{dim} \mathfrak{h}(\mathfrak{s})$ so that $\ell(\mathfrak{s})$ is the rank of $\mathfrak{s}$ and

$$
\ell=\ell(\mathfrak{t})+\ell(\mathfrak{s})
$$


Let $\Pi=\left\{\alpha_{1}, \ldots, \alpha_{\ell}\right\}$ be the set of simple positive roots in $\Delta_{+}$. If $\varphi \in \Delta_{+}(\mathfrak{s})$ and $\varphi=\varphi_{1}+\varphi_{2}$ where $\varphi_{1}, \varphi_{2} \in \Delta_{+}$, obviously $\left(\varphi_{1}\right)_{\mathfrak{t}}=-\left(\varphi_{2}\right)_{\mathfrak{t}}$. But then $\left(\varphi_{i}\right)_{\mathfrak{t}}, i=1,2$, vanish by Lemma 2.4 so that $\varphi_{1}, \varphi_{2} \in \Delta_{+}(\mathfrak{s})$. Hence if $\varphi \in \Delta_{+}(\mathfrak{s})$ is simple with respect to $\Delta_{+}(\mathfrak{s})$, it is simple with respect to $\Delta_{+}$. We may therefore order $\Pi$ so that $\alpha_{\ell(\mathfrak{t})+i} \in \Delta_{+}(\mathfrak{s})$, for $i=1 \ldots, \ell(\mathfrak{s})$, and hence if $\Pi_{\mathfrak{s}}=\left\{\alpha_{\ell(\mathfrak{t})+1}, \ldots, \alpha_{\ell}\right\}$, then

$$
\Pi_{\mathfrak{s}} \text { is a basis of } \mathfrak{h}(\mathfrak{s})^{*} ; \text { see }(1.6) \text {. }
$$

A $\mathfrak{t}$-root $\nu \in R_{+}$is called simple if $\nu$ cannot be written $\nu=\nu_{1}+\nu_{2}$ where $\nu_{1}, \nu_{2} \in R_{+}$. Let $R_{\text {simp }}$ be the set of simple $\mathfrak{t}$-roots in $R_{+}$.

Lemma 2.6. Assume $\xi_{1}, \xi_{2} \in R_{\text {simp }}$ are distinct. Then

$$
\left(\xi_{1}, \xi_{2}\right) \leq 0
$$

so that, by Remark 2.5, the elements in $R_{\text {simp }}$ are linearly independent. In particular

$$
\operatorname{card} R_{\text {simp }} \leq \ell(\mathfrak{t})
$$

Proof. Assume $\left(\xi_{1}, \xi_{2}\right)>0$. Then, by Theorem 2.3, $\xi_{1}-\xi_{2}$ and $\xi_{2}-\xi_{1}$ are in $R$. Without loss assume $\nu \in R_{+}$where $\nu=\xi_{1}-\xi_{2}$. Then $\xi_{1}=\nu+\xi_{2}$. This contradicts the simplicity of $\xi_{1}$. Hence one has (2.28). QED

As noted in (1.6), $\mathfrak{h}(\mathfrak{s})^{*}$ is the orthocomplement of $\mathfrak{t}$ in $\mathfrak{h}$. Thus, by (2.27), if

$$
\beta_{j}=\left(\alpha_{j}\right)_{\mathfrak{t}}
$$

for $j=1, \ldots, \ell(\mathfrak{t})$, then clearly

$$
\begin{aligned}
& \beta_{j} \in R_{+} \text {and } \\
& \beta_{j}, j=1, \ldots, \ell(\mathfrak{t}), \text { are a basis of } \mathfrak{t}_{\mathbb{R}}^{*} .
\end{aligned}
$$

Recalling (1.1), for any $\nu \in R$ and $j=1, \ldots, \ell(\mathfrak{t})$, let $n_{j}(\nu)=n_{\alpha_{j}}(\varphi)$ where $\varphi \in \Delta\left(\mathfrak{g}_{\nu}\right)$. This is independent of the choice of $\varphi$ by $(2.27)$.

Theorem 2.7. One has

$$
R_{\text {simp }}=\left\{\beta_{1}, \ldots, \beta_{\ell(\mathfrak{t})}\right\}
$$


so that $R_{\mathrm{simp}}$ is a basis of $\mathfrak{t}_{\mathbb{R}}^{*}$ and for $i \neq j$,

$$
\left(\beta_{i}, \beta_{j}\right) \leq 0
$$

Furthermore for $\nu \in R_{+}$and $j=1, \ldots, \ell(\mathfrak{t})$, one has

$$
\nu=\sum_{j=1}^{\ell(\mathfrak{t})} n_{j}(\nu) \beta_{j} .
$$

Proof. Let $j \in\{1, \ldots, \ell(\mathfrak{t})\}$. Assume that $\beta_{j} \notin R_{\text {simp. Then there exists }}$ $\nu_{1}, \nu_{2} \in R_{+}$such that $\beta_{j}=\nu_{1}+\nu_{2}$. But then by (3) in Theorem 2.3 one has

$$
\left[\mathfrak{g}_{\nu_{1}}, \mathfrak{g}_{\nu_{2}}\right]=\mathfrak{g}_{\beta_{j}}
$$

But $e_{\alpha_{j}} \in \mathfrak{g}_{\beta_{j}}$ by (1.23). Thus there exist, for $i=1,2, \varphi_{i} \in \Delta\left(\mathfrak{g}_{\nu_{i}}\right)$ such that $\varphi_{1}+\varphi_{2}=\alpha_{j}$. This contradicts the simplicity of $\alpha_{j}$ since $\varphi_{1}, \varphi_{2} \in \Delta_{+}$. But then (2.32) follows from (2.29). Also (2.33) and the fact that $R_{\text {simp }}$ is a basis of $\mathfrak{t}_{\mathbb{R}}^{*}$ follow from Lemma 2.6.

Now let $\nu \in R_{+}$and let $\varphi \in \Delta\left(\mathfrak{g}_{\nu}\right)$. Recalling the expansion (1.1) one immediately has

$$
\nu=\sum_{\alpha \in \Pi} n_{\alpha}(\varphi) \alpha_{\mathfrak{t}}
$$

but this yields (2.34). QED

Remark 2.8. Note that if $j=1, \ldots, \ell(\mathfrak{t})$, one has that $e_{\alpha_{j}} \in \mathfrak{g}_{\beta_{j}}$ and $e_{\alpha_{j}}$ is a lowest weight vector for the irreducible $\mathfrak{m}$-module $\mathfrak{g}_{\beta_{j}}$. Indeed this is clear since for $i=1, \ldots, \ell(\mathfrak{s})$,

$$
\left[e_{-\alpha_{\ell(\mathfrak{t})+1}}, e_{\alpha_{j}}\right]=0
$$

2.4. Henceforth we will assume that $\mathfrak{g}$ is simple. Let $\psi \in \Delta_{+}$be the highest root so that, by the simplicity of $\mathfrak{g}$,

$$
\text { cent } \mathfrak{n}_{\mathfrak{b}}=\mathbb{C} e_{\psi}
$$

where we recall $(\S 1.1) \mathfrak{n}_{\mathfrak{b}}=[\mathfrak{b}, \mathfrak{b}]$.

Remark 2.9. For any $\alpha \in \Pi$ one knows that

$$
n_{\alpha}(\psi)>0
$$


and, for any $\varphi \in \Delta_{+}$,

$$
n_{\alpha}(\psi) \geq n_{\alpha}(\varphi)
$$

Indeed (2.39) and (2.40) are consequences of the immediate fact that

$$
e_{\psi} \in U\left(\mathfrak{n}_{\mathfrak{b}}\right) e_{\varphi}
$$

where any Lie algebra $\mathfrak{a}, U(\mathfrak{a})$ is the enveloping algebra of $\mathfrak{a}$.

$$
\begin{aligned}
& \text { For any } \nu \in R \text { let } o(\nu)=\sum_{j=1}^{\ell(\mathfrak{t})} n_{\beta_{j}}(\nu) \text {, and for any } k \in \mathbb{Z}_{+} \text {let } \\
& \qquad \mathfrak{n}(k)=\sum_{\nu \in R, o(\nu)=k} \mathfrak{g}_{\nu} .
\end{aligned}
$$

For $j, k \in \mathbb{Z}$, clearly

$$
[\mathfrak{n}(j), \mathfrak{n}(k)] \subset \mathfrak{n}(j+k) .
$$

Now $\psi_{\mathfrak{t}} \neq 0$ by Remark 2.9 (one has $\ell(\mathfrak{t})<\ell$ by our assumption in $\S 1.1$ ). Let $\nu($ cent $) \in R$ be defined by putting $\nu($ cent $)=\psi_{\text {t }}$. Put $k($ cent $)=o(\nu($ cent $))$ so that

$$
k(\text { cent })=\sum_{i=1}^{\ell(\mathfrak{t})} n_{\alpha_{i}}(\psi) .
$$

Remark 2.9 clearly also implies

Proposition 2.10. $\mathfrak{n}(k)=0$ if $k>k$ (cent) and

$$
\mathfrak{n}(k(\text { cent }))=\mathfrak{g}_{\nu(\text { cent })}
$$

Furthermore one has the direct sum

$$
\mathfrak{n}=\sum_{k=0}^{k(\text { cent })} \mathfrak{n}(k)
$$

The upper central series of $\mathfrak{n}$ (defined for any nilpotent Lie algebra) is a sequence of distinct ideals $\mathfrak{n}_{1} \subset n_{2} \subset \cdots \subset \mathfrak{n}_{d}=\mathfrak{n}$ where $\mathfrak{n}_{1}=$ cent $\mathfrak{n}$ and for $i \geq 2$,

$$
n_{i} / \mathfrak{n}_{i-1}=\operatorname{cent} \mathfrak{n} / \mathfrak{n}_{i-1}
$$

See (14) on p. 29 in [Jac]. We refer to $d$ as the length of the upper central series. 
Theorem 2.11. If $i<k($ cent $)$ and $\nu \in \mathfrak{n}(i)$, there exists $j \in\{1, \ldots, \ell(\mathfrak{t})\}$ such that $\nu+\beta_{j} \in \mathfrak{n}(i+1)$ so that

$$
\left[\mathfrak{g}_{\nu}, \mathfrak{g}_{\beta_{j}}\right]=\mathfrak{g}_{\nu+\beta_{j}}
$$

In particular $\left[\mathfrak{g}_{\nu}, \mathfrak{g}_{\beta_{j}}\right] \neq 0$. Furthermore

$$
\mathfrak{g}_{\nu(\text { cent })}=\operatorname{cent} \mathfrak{n}
$$

and the upper central series $\mathfrak{n}_{i}$, of the nilradical $\mathfrak{n}$ of the general proper parabolic subalgebra $\mathfrak{q}$, is given as follows:

$$
\mathfrak{n}_{i}=\sum_{j=1}^{i} \mathfrak{n}(k(\text { cent })-j+1)
$$

noting that $k$ (cent) is the length of the upper central series.

Proof. Let $\varphi \in \Delta\left(\mathfrak{g}_{\nu}\right)$ be the highest weight of the $\mathfrak{m}$-irreducible module $\mathfrak{g}_{\nu}$. Thus $\left[e_{\alpha_{i+\ell(\mathfrak{t})}}, e_{\varphi}\right]=0$ for $i=1, \ldots, \ell(\mathfrak{s})$. But $\varphi \neq \psi$. Hence by the uniquenss of the $\mathfrak{g}-$ highest weight there must exist $j=1, \ldots, \ell(\mathfrak{t})$ such that $\left[e_{\alpha_{j}}, e_{\varphi}\right] \neq 0$. But this implies (2.48). Now obviously $\mathfrak{g}_{\nu \text { (cent) }} \subset$ cent $\mathfrak{n}$ by (2.43) and (2.46). But clearly cent $\mathfrak{n}$ is stable under ad $\mathfrak{m}$. Therefore to prove (2.49) it suffices to show that $\mathfrak{g}_{\nu} \not \subset$ cent $\mathfrak{n}$ for $\nu \neq \nu$ (cent). But this is established by (2.48). Let $i>1$. Assume inductively that one has (2.50) where $i-1$ replaces $i$. Then, returning to (2.50) as stated, if $\mathfrak{n}_{(i)}$ is given by the right side of (2.50), one has $\mathfrak{n}_{(i)} \subset \mathfrak{n}_{i}$ by (2.43) and (2.46). But the upper central series (e.g., by induction) is stabilized by ad $\mathfrak{m}$ so that in particular $\mathfrak{n}_{i}$ is stabilized by ad $\mathfrak{m}$. But again (2.48) implies that if $\mathfrak{g}_{\nu} \subset \mathfrak{n}(j)$ where $j<k($ cent $)-i+1$, then $\mathfrak{g}_{\nu} \not \subset \mathfrak{n}_{i}$. This implies that $\mathfrak{n}_{i}=\mathfrak{n}_{(i)}$. QED

Using the notation in [Jac], see page 23 , the lower central series $\mathfrak{n}^{i}$ of $\mathfrak{n}$ is a sequence of ideals defined inductively so that $\mathfrak{n}^{1}=\mathfrak{n}$ and for $i>1, \mathfrak{n}^{i}=\left[\mathfrak{n}, \mathfrak{n}^{i-1}\right]$. See also p. 11 in [Hum]. The indexing in [Hum] differs by 1 from the indexing in [Jac]. We will call the maximum $k$ such that $\mathfrak{n}^{k} \neq 0$ the length of the lower central series.

Theorem 2.12. Let $i$ be any integer where $2 \leq i \leq k$ (cent) and let $\nu \in R$ where $\mathfrak{g}_{\nu} \in \mathfrak{n}(i)$. Then there exists $j \in\{1, \ldots, \ell(\mathfrak{t})\}$ and $\mu \in R$ where $\mathfrak{g}_{\mu} \in \mathfrak{n}(i-1)$ such that

$$
\left[\mathfrak{g}_{\beta_{j}}, \mathfrak{g}_{\mu}\right]=\mathfrak{g}_{\nu}
$$


so that

$$
[\mathfrak{n}(1), \mathfrak{n}(i-1)]=\mathfrak{n}(i)
$$

In particular the lower central series $\mathfrak{n}^{i}$ of the nilradical $\mathfrak{n}$ of the arbitrary proper parabolic subalgebra $\mathfrak{q}$ of $\mathfrak{g}$ is given by

$$
\mathfrak{n}^{i}=\sum_{j=i}^{k(\text { cent })} n(j)
$$

so that (see 2.44) $k$ (cent) is the length of the lower central series of $\mathfrak{n}$ (as well as the upper central series, see Theorem 2.11). The lower and upper central series of $\mathfrak{n}$ are therefore, except for indexing, the same

$$
\mathfrak{n}^{i}=\mathfrak{n}_{k(\text { cent })-i+1}
$$

for $i=1, \ldots, k$ (cent).

Proof. It suffices only to prove (2.51). But, by Remark 2.5 and Theorem 2.7, $\beta_{j} \in R_{\text {simp }}$ exists so that

$$
\left(\nu, \beta_{j}\right)>0 .
$$

Thus $\mu \in R$ where $\mu=\nu-\beta_{j}$ by Theorem 2.3. But also necessarily $n_{j}(\nu)>0$ by (2.33) so that $\mathfrak{g}_{\mu} \in \mathfrak{n}(i-1)$. But $\mu+\beta_{j}=\nu$. Thus by (3) of Theorem 2.3 one has (2.51). QED

\section{Borel-de Siebenthal theory, special elements, and the Lie subalgebras they define}

3.1. We continue to assume (starting in $\S 2.4$ ) that $\mathfrak{g}$ is simple. In this section we will apply the results of $\S 1$ and $\S 2$ to the case where $\ell(\mathfrak{t})=1$. It will be convenient to change some notation and earlier indexing. In particular we now fix an ordering in $\Pi$ so that $\Pi=\left\{\alpha_{1}, \ldots, \alpha_{\ell}\right\}$. Also recalling (1.1) we will write $n_{i}(\varphi)$ for $n_{\alpha_{i}}(\varphi)$ so that for the highest root $\psi$ one has

$$
\psi=\sum_{i=1}^{\ell} n_{i}(\psi) \alpha_{i} .
$$

Now let $x_{j}, j=1, \ldots, \ell$, be the basis of $\mathfrak{h}_{\mathbb{R}}$ so that

$$
\left\langle\alpha_{l}, x_{j}\right\rangle=\delta_{i j}
$$


Now for $j=1, \ldots, \ell$, let $\mathfrak{q}[j]$ be the maximal standard parabolic subalgebra defined by $x_{j}$. The standard Levi factor $\mathfrak{m}[j]$ of $\mathfrak{q}[j]$ is the centralizer $\mathfrak{g}^{x_{j}}$ of $x_{j}$ in $\mathfrak{g}$. The decomposition (1.3) now becomes

$$
\mathfrak{m}[j]=\mathfrak{t}[j]+\mathfrak{s}[j]
$$

where the one-dimensional center $\mathfrak{t}[j]$ of $\mathfrak{m}[j]$ is given by

$$
\mathfrak{t}[j]=\mathbb{C} x_{j} .
$$

If $\Pi_{\mathfrak{s}[j]}$ is the set of simple positive roots of the (rank $\left.\ell-1\right)$-semisimple Lie algebra $\mathfrak{s}[j]$, defined as in $(2.27)$, one now has

$$
\Pi_{\mathfrak{s}[j]}=\Pi \backslash\left\{\alpha_{j}\right\}
$$

The nilradical $\mathfrak{n}[j]$ of $\mathfrak{q}[j]$ is given by

$$
\mathfrak{n}[j]=\operatorname{span} \text { of }\left\{e_{\varphi} \mid n_{j}(\varphi)>0\right\}
$$

Write $R[j]$ for $R, R[j]_{+}$for $R_{+}$and $R[j]_{\text {simp }}$ for $R_{\text {simp }}$. Let $\beta[j]=\left(\alpha_{j}\right)_{\mathfrak{t}[j]}$ so that

$$
\left\langle\beta[j], x_{j}\right\rangle=1 \text {. }
$$

Proposition 3.1. One has

$$
R[j]_{\operatorname{simp}}=\{\beta[j]\}
$$

and

$$
R[j]_{+}=\left\{\beta[j], 2 \beta[j], \ldots, n_{j}(\psi) \beta[j]\right\}
$$

and

$$
R[j]=\left\{ \pm \beta[j], \pm 2 \beta[j], \ldots, \pm n_{j}(\psi) \beta[j]\right\}
$$

In particular

$$
\begin{aligned}
\operatorname{card} R[j]_{+} & =n_{j}(\psi) \\
\operatorname{card} R[j] & =2 n_{j}(\psi) .
\end{aligned}
$$

Proof. The proof is immediate from (2.17), (2.25), Theorem 2.7, Theorem 2.12 and (2.44) which implies here that

$$
k(\text { cent })=n_{j}(\psi)
$$


Let $I[j]=\left\{ \pm 1, \ldots, \pm n_{j}(\psi)\right\}$ so that if $\nu \in R[j]$, then $\nu=k \beta[j]$ for $k \in I[j]$. We recall (see $\S 1.2$ ) that for $k \in I[j]$,

$$
\begin{aligned}
\mathfrak{g}_{k \beta[j]} & =\text { span of }\left\{e_{\varphi} \mid n_{j}(\varphi)=k\right\} \\
& =\left\{z \in \mathfrak{g} \mid\left[x_{j}, z\right]=k z\right\}
\end{aligned}
$$

One has the direct sums

$$
\begin{aligned}
\mathfrak{n}[j] & =\sum_{k=1}^{n_{j}(\psi)} \mathfrak{g}_{k \beta[j]} \\
\mathfrak{g} & =\mathfrak{m}[j]+\sum_{k \in I[j]} \mathfrak{g}_{k \beta[j]} .
\end{aligned}
$$

One now has

Theorem 3.2. Let $j \in\{1, \ldots, \ell\}$. Then $\mathfrak{g}_{k \beta[j]}$ is ad $\mathfrak{m}[j]$-irreducible for any $k \in$ $I[j]$. In particular the nilradical $\mathfrak{n}[j]$ of $\mathfrak{q}[j]$ is, as an ad $\mathfrak{m}[j]$-module, multiplicity-free with $n_{j}(\psi)$-irreducible components. Also $\mathfrak{g} / \mathfrak{m}[j]$ is, as an $\operatorname{ad} \mathfrak{m}[j]$-module, multiplicityfree with $2 n_{j}(\psi)$-irreducible components. Finally, if $p, q \in I[j]$ and $p+q \in I[j]$, then

$$
\left[\mathfrak{g}_{p \beta[j]}, \mathfrak{g}_{q \beta[j]}\right]=\mathfrak{g}_{(p+q) \beta[j]} .
$$

Proof. The first statement is just the present application of Theorem 1.9. The equality (3.15) is given by (3) of Theorem 2.3. QED

3.2. Let $C \subset \mathfrak{h}_{\mathbb{R}}$ be the fundamental Weyl chamber corresponding to $\mathfrak{b}$ so that

$$
C=\left\{x \in \mathfrak{h}_{\mathbb{R}} \mid\left\langle\alpha_{i}, x\right\rangle \geq 0, \text { for } i=1, \ldots, \ell\right\},
$$

and let $A \subset C$ be the fundamental alcove so that $A$ is the simplex defined by

$$
A=\{x \in C \mid\langle\psi, x\rangle \leq 1\}
$$

Let $G$ be a simply-connected complex group for which $\mathfrak{g}=$ Lie $G$. Let $K$ be a maximal compact subgroup of $G$. We may choose $K$ so that if $\mathfrak{k}=$ Lie $K$, then $i h_{\mathbb{R}}=$ Lie $H_{\text {comp }}$ where $H_{\text {comp }}$ is a maximal torus of $K$. 
A classical result of Cartan and Weyl is the statement

Proposition 3.3. For any element $g \in K$ there exists a unique element $x \in A$ such that

$$
g \text { is } K \text {-conjugate to } \exp 2 \pi i x \text {. }
$$

Clearly the $\ell+1$ vertices $v_{j}, j=0, \ldots, \ell$, of $A$ are then given as $v_{0}=0$ and for $j>0$,

$$
v_{j}=x_{j} / n_{j}(\psi) .
$$

For $j=1, \ldots, \ell$, let $a_{j} \in K$ be defined by putting

$$
a_{j}=\exp 2 \pi i v_{j}
$$

Let $\omega[j]$ be the $n_{j}(\psi)$-root of unity given by putting $\omega[j]=e^{2 \pi i / n_{j}(\psi)}$. For $g \in G$ let $G^{g}$ (resp. $\mathfrak{g}^{g}$ ) be the centralizer of $g$ in $G$ (resp. $\mathfrak{g}$ ). As an immediate consequence of (3.14) the well-known adjoint action of $a_{j}$ on $\mathfrak{g}$ is given by

Proposition 3.4. Ad $a_{j}$ has order $n_{j}(\psi)$ on $\mathfrak{g}$. In fact one has

$$
\begin{aligned}
\operatorname{Ad} a_{j} & =1 \text { on } \mathfrak{m}[j] \\
& =\omega[j]^{k} \text { on } \mathfrak{g}_{k \beta[j]} \text { for all } k \in I[j] .
\end{aligned}
$$

In particular one has the direct sum

$$
\mathfrak{g}^{a_{j}}=\mathfrak{m}[j]+\mathfrak{g}_{n_{j}(\psi) \beta[j]}+\mathfrak{g}_{-n_{j}(\psi) \beta[j]}
$$

An element $a \in K$ is called special if the reductive subalgebra $\mathfrak{g}^{a}$ of $\mathfrak{g}$ is in fact semisimple (i.e., cent $\mathfrak{g}^{a}=0$ ). The following is also well known but proved here for completeness.

Proposition 3.5. For $j=1, \ldots, \ell$, the element $a_{j}$ is special.

Proof. Since $\mathfrak{h} \subset \mathfrak{m}[j]$ one obviously has cent $\mathfrak{g}^{a_{j}} \subset \operatorname{cent} \mathfrak{m}[j]$. But cent $\mathfrak{m}[j]=$ $\mathbb{C} x_{j}$. However ad $x_{j}=n_{j}(\psi)$ on $\mathfrak{g}_{n_{j}(\psi) \beta[j]}$. Thus cent $\mathfrak{g}^{a_{j}}=0$. QED

Remark 3.6. One can readily prove $a \in K$ is special if and only if either $a=1$ or there exists $j \in\{1, \ldots, \ell\}$, necessarily unique, such that $a$ is $K$-conjugate to $a_{j}$. 
We also remark that special elements arise in connection with distinguished nilpotent conjugacy classes in $\mathfrak{g}$. Indeed if $e$ is a distinguished nilpotent element then, where $G_{o}^{e}$ is the identity component of $G^{e}$, the component group $G^{e} / G_{o}^{e}$ is isomorphic to the finite group $F$ where $F$ is the centralizer in $G$ of a TDS containing $e$. Since $F$ is finite we may make choices so that $F \subset K$. But then the elements of $F$ are special. Indeed if $a \in F$, then $e \in \mathfrak{g}^{a}$. But if $x \in \operatorname{cent} \mathfrak{g}^{a}$, then $x$ is a semisimple element that commutes with $e$. Thus $x=0$ since $e$ is distinguished. Hence cent $\mathfrak{g}^{a}=0$.

3.3. Let $j=1, \ldots, \ell$. For completeness in this section we wish to give the proof of the Borel-de Siebenthal algorithm for determining the Dynkin diagram of $\mathfrak{g}^{a_{j}}$. We recall that the extended Dynkin diagram of $\mathfrak{g}$ is the usual Dynkin diagram (whose nodes are identified with $\Pi$ ) of $\mathfrak{g}$, together with an additional node $\alpha_{0}$, where if $i=1, \ldots, \ell$, then $\alpha_{0}$ is linked to $\alpha_{i}$ with

$$
m_{i}=2\left(\alpha_{i}, \psi\right) /\left(\alpha_{i}, \alpha_{i}\right)
$$

lines and arrowhead at $\alpha_{i}$, directed at $\alpha_{i}$, if $m_{i}>1$.

Theorem 3.7. (Borel-de Siebenthal algorithm). The Dynkin diagram of $\mathfrak{g}^{a_{j}}$ is the subdiagram of the extended Dynkin diagram remaining after deleting $\alpha_{j}$ (and all lines linked to $\alpha_{j}$ ) from the extended Dynkin diagram.

Proof. Let $\mathfrak{m}[j]_{+}=\mathfrak{m}[j] \cap \mathfrak{b}$ so that $\mathfrak{m}[j]_{+}$is a Borel subalgebra, containing $\mathfrak{h}$, of $\mathfrak{m}[j]$. Now $\mathfrak{g}_{-n_{j}(\psi) \beta[j]}$ is clearly a commutative nilpotent subalgebra of $\mathfrak{g}^{a_{j}}$ which is stable (and in fact irreducible) under $\operatorname{ad} \mathfrak{m}[j]$. But then of course $\mathfrak{g}_{-n_{j}(\psi) \beta[j]}$ is stable under ad $\mathfrak{m}[j]_{+}$. Thus

$$
\mathfrak{b}[j]=\mathfrak{m}[j]_{+}+\mathfrak{g}_{-n_{j}(\psi) \beta[j]}
$$

is a solvable Lie subalgebra of $\mathfrak{g}^{a_{j}}$. But, by dimension, $\mathfrak{b}[j]$ is then a Borel subalgebra of $\mathfrak{g}^{a_{j}}$. Regarding $\Delta(\mathfrak{b}[j])$ as a system of positive roots for $\mathfrak{g}^{a_{j}}$, let $\Pi[j]$ be the corresponding set of simple positive roots. But now $-\psi \in \Delta\left(\mathfrak{g}_{-n_{j}(\psi) \beta[j]}\right)$. (In fact clearly $e_{-\psi}$ is a lowest weight vector for the irreducible action of $\operatorname{ad} \mathfrak{m}[j]$ on $\mathfrak{g}_{-n_{j}(\psi) \beta[j]}$.) But one notes it is immediate that $-\psi \in \Pi[j]$. Since $\mathfrak{g}^{a_{j}}$ has rank $\ell$ one has, recalling (3.5),

$$
\Pi[j]=\Pi_{\mathfrak{s}[j]} \cup\{-\psi\} .
$$

But then the Dynkin diagram of $\mathfrak{g}^{a_{j}}$ is the Dynkin diagram of $\mathfrak{s}[j]$, together with the node defined by $-\psi$, where if $\alpha_{i} \in \Pi_{\mathfrak{s}[j]}$, then $-\psi$ (a long root) is linked to $\alpha_{i}$ by 
(see (3.23)) $m_{i}$ lines and arrowhead at $\alpha_{i}$, directed at $\alpha_{i}$, if $m_{i}>1$. But this is the Borel-de Siebenthal algorithm. QED

3.4. Let $j=1, \ldots, \ell$, and let $I_{o}[j]=I[j] \backslash\left\{ \pm n_{j}(\psi)\right\}$. Let

$$
\mathfrak{r}[j]=\sum_{k \in I_{o}[j]} \mathfrak{g}_{k \beta[j]}
$$

so that by (3.14) and (3.22) one has the $B$-orthogonal direct sum

$$
\mathfrak{g}=\mathfrak{g}^{a_{j}}+\mathfrak{r}[j]
$$

For $k=1, \ldots, n_{j}(\psi)-1$, let

$$
\mathfrak{g}\left[a_{j}\right]^{k}=\left\{z \in \mathfrak{g} \mid \operatorname{Ad} a_{j}(z)=\omega[j]^{k} z\right\}
$$

Obviously

$$
\mathfrak{g}\left[a_{j}\right]^{k} \text { is stable under ad } \mathfrak{g}^{a_{j}} \text { for } k=1, \ldots, n_{j}(\psi)-1 .
$$

But clearly, by Proposition 3.4, for $k=1, \ldots, n_{j}(\psi)-1$,

$$
\mathfrak{g}\left[a_{j}\right]^{k}=\mathfrak{g}_{k \beta[j]}+\mathfrak{g}_{\left(k-n_{j}(\psi)\right) \beta[j]},
$$

and hence by (3.26) one has the direct sum

$$
\mathfrak{r}[j]=\sum_{k=1}^{n_{j}(\psi)-1} \mathfrak{g}\left[a_{j}\right]^{k}
$$

Consequently one notes that not only is $\mathfrak{r}[j]$ stable under ad $g^{a_{j}}$ but (3.31) isolates $n_{j}(\psi)-1$ ad $g^{a_{j}}$-submodules of $\mathfrak{r}[j]$.

Theorem 3.8. Let $j=1, \ldots, j$, so that $a_{j}$, defined by the vertex $v_{j}$ of the fundamental alcove, is a special element of $K$. In particular, its centralizer $\mathfrak{g}^{a_{j}}$ is a maximal semisimple Lie subalgebra of $\mathfrak{g}$ if $n_{j}(\psi)$ is prime-by Borel-de Siebenthal theory. Let $\mathfrak{r}[j]$ be the B-orthocomplement of $\mathfrak{g}^{a_{j}}$ in $\mathfrak{g}$. Then $\mathfrak{r}[j]$ is a multiplicity-free $\operatorname{ad} \mathfrak{m}[j]$-module where $\mathfrak{m}[j] \subset \mathfrak{g}^{a_{j}}$ is the centralizer of $v_{j}$ in $\mathfrak{g}$ and (3.26) is the decomposition of $r[j]$ into a sum of $2\left(n_{j}(\psi)-1\right)$-irreducible ad $\mathfrak{m}[j]$-submodules. Next (3.30) defines the decomposition of the $\operatorname{Ad} a_{j}$-weight space $\mathfrak{g}\left[a_{j}\right]^{k}$ into a sum of two irreducible ad $m[j]$-submodules. Furthermore, and mainly, the Ad $a_{j}$-weight space $\mathfrak{g}\left[a_{j}\right]^{k}$ 
is an irreducible $\mathrm{ad}_{\mathfrak{g}} \mathfrak{g}^{a_{j}}$-submodule. In addition $\mathfrak{r}[j]$ is a multiplicity-free $\mathrm{Ad}_{\mathfrak{g}} \mathfrak{g}^{a_{j}}$-module and (3.31) is the decomposition of $r[j]$ into a sum of $n_{j}(\psi)-1$-irreducible ad $\mathfrak{g}^{a_{j}}$ submodules. Finally, if $p, q, r \in\left\{1, \ldots, n_{j}(\psi)-1\right\}$ and $r \cong p+q \bmod n_{j}(\psi)$, then

$$
\left[\mathfrak{g}\left[a_{j}\right]^{p}, \mathfrak{g}\left[a_{j}\right]^{q}\right]=\mathfrak{g}\left[a_{j}\right]^{r}
$$

Proof. Up until the sentence beginnning with "Furthermore" the stated results have been established in Theorem 3.2. But now

$$
\left[\mathfrak{g}_{\left(k-n_{j}(\psi)\right) \beta[j]}, \mathfrak{g}_{n_{j}(\psi) \beta[j]}\right]=\mathfrak{g}_{k \beta[j]}
$$

and

$$
\left[\mathfrak{g}_{k \beta[j]}, \mathfrak{g}_{-n_{j}(\psi) \beta[j]}\right]=\mathfrak{g}_{\left(k-n_{j}(\psi)\right) \beta[j]}
$$

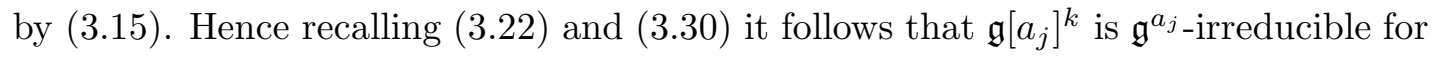
all $k=1, \ldots, n_{j}(\psi)-1$.

Clearly the left side of (3.32) is contained in the right side of (3.32). But by ad $g^{a_{j}}$ irreducibility one has (3.32) as soon as one observes that the left side is nonzero. But this is clear from (3.15) if $p+q=r$. If $p+q>n_{j}(\psi)$, then $r=p+q-n_{j}(\psi)$. But then (3.32) follows from (3.15) where $q$ is replaced by $q-n_{j}(\psi)$. QED

Remark 3.9. One of the main results of Borel-de Siebenthal theory is the statement that $\mathfrak{g}_{1}$ is a maximal proper (i.e., $g_{1} \neq \mathfrak{g}$ ) semisimple subalgebra such that $\operatorname{rank} \mathfrak{g}_{1}=\operatorname{rank} \mathfrak{g}$ if and only if

$$
\mathfrak{g}_{1} \cong \mathfrak{g}^{a_{j}}
$$

where $n_{j}(\psi)$ is a prime number. This may be proved as follows: In one direction assume (3.33) where $n_{j}(\psi)$ is prime. Then if $\mathfrak{g}_{o}$ is a subalgebra where $\mathfrak{g}^{a_{j}} \subset \mathfrak{g}_{o}$ and $\mathfrak{g}_{o} \neq \mathfrak{g}^{a_{j}}$, there must exist, by (3.27), $0 \neq x \in \mathfrak{r}[j] \cap \mathfrak{g}_{o}$. But since $\mathfrak{g}_{o}$ is stable under ad $\mathfrak{g}^{a_{j}}$ it follows from Theorem 3.8 that there exists $k \in\left\{1, \ldots, n_{j}(\psi)-1\right\}$ such that $\mathfrak{g}\left[a_{j}\right]^{k} \subset \mathfrak{g}_{o}$. But $\mathfrak{g}$ is generated by $\mathfrak{g}^{a_{j}}$ and $\mathfrak{g}\left[a_{j}\right]^{k}$, by $(3.32)$, since $n_{j}(\psi)$ is prime. Thus $\mathfrak{g}^{a_{j}}$ is maximal as a proper Lie subalgebra of $\mathfrak{g}$. Conversely assume $\mathfrak{g}_{1}$ is a maximal proper semisimple subalgebra where $\operatorname{rank} \mathfrak{g}_{1}=\operatorname{rank} \mathfrak{g}$. Let $G_{1} \subset G$ be the subgroup corresponding to $\mathfrak{g}_{1}$. Let $\gamma$ denote the adjoint representation of $G_{1}$ on $\mathfrak{g} / \mathfrak{g}_{1}$. By the equal rank condition 0 is not a weight of $\gamma$. Thus $\gamma$ does not descend to the adjoint group of $\mathfrak{g}_{1}$. Thus there exists $1 \neq c \in \operatorname{cent} G_{1}$ such that $c \notin \operatorname{Ker} \gamma$. But $c$ has 
finite order since $\mathfrak{g}_{1}$ is semisimple. We may therefore make choices so that $c \in K$. Of course $\mathfrak{g}_{1} \subset \mathfrak{g}^{c}$. By maximality

$$
\mathfrak{g}^{c}=\mathfrak{g}_{1}
$$

But then $c$ is special and by Remark 3.6 choices can be made so that $c=a_{j}$ for some $j \in\{1, \ldots, \ell\}$. But if $n_{j}(\psi)$ is not prime there exists an integer $1<k<n_{j}(\psi)$ such that $k$ divides $n_{j}(\psi)$. But then, by (3.32), $\mathfrak{g}_{1}$ and $\mathfrak{g}\left[a_{j}\right]^{k}$ generate a proper semisimple subalgebra of $\mathfrak{g}$, contradicting the maximality of $\mathfrak{g}_{1}$.

\section{Example}

4.1. In this section we consider the example of the theory above for the case where, for a positive integer $n>1, \mathfrak{g}=\operatorname{Lie} S l(n, \mathbb{C})$. With the usual meaning of matrix units, $e_{i j}, x \in \mathfrak{g}$, when we can write

$$
x=\sum_{i, j=1}^{n} a_{i j}(x) e_{i j}
$$

where $\sum_{i=1}^{n} a_{i i}(x)=0$. For $k$ a positive integer, where $1<k \leq n$, let

$$
\delta=\left\{d_{1}, \ldots, d_{k}\right\}
$$

where the $d_{p}$ are positive integers such that

$$
\sum_{p=1}^{k} d_{p}=n
$$

For $q \in\{1, \ldots, k\}$, put

$$
f_{q}=\sum_{p=1}^{q} d_{p}
$$

and hence

$$
1 \leq f_{1}<\cdots<f_{k}=n
$$

Now for any $i \in\{1, \ldots, n\}$, let $q(i) \in\{1, \ldots, k\}$ be the minimum value of $q$ such that $i \leq f_{q}$. Thus if we put $f_{0}=0$, and we let $I_{q}$ be the half-open interval of integers given by putting $I_{q}=\left(f_{q-1}, f_{q}\right]$, then one has the disjoint union

$$
(0, n]=\sqcup_{q=1}^{k} I_{q}
$$


Clearly one has

$$
\operatorname{card} I_{q}=d_{q}
$$

and for any $i \in(0, n]$ one has

$$
i \in I_{q(i)} \text {. }
$$

Next put

$$
\mathfrak{n}(\delta)=\left\{x \in \mathfrak{g} \mid a_{i j}(x)=0, \text { unless } j>f_{q(i)}\right\} .
$$

In addition for $r, s \in\{1, \ldots, q\}$, where $r \neq s$ let

$$
\mathfrak{g}_{r, s}(\delta)=\left\{x \in \mathfrak{g} \mid a_{i j}(x)=0 \text {, unless } i \in I_{r} \text {, and } j \in I_{s}\right\}
$$

One readily notes that $\mathfrak{n}(\delta)$ is a nilpotent Lie algebra and one has the vector space direct sum

$$
\mathfrak{n}(\delta)=\oplus_{r<s} \mathfrak{g}_{r, s}(\delta) .
$$

Let $\overline{\mathfrak{n}}(\delta)$ be the transpose of $\mathfrak{n}(\delta)$. One then has the direct sum

$$
\overline{\mathfrak{n}}(\delta)=\oplus_{s<r} \mathfrak{g}_{r, s}(\delta)
$$

Also for $q \in\{1, \ldots, k\}$, let

$$
\mathfrak{s}_{q}(\delta)=\left\{x \in \mathfrak{g} \mid a_{i j}(x)=0, \text { unless } i, j \in I_{q}\right\}
$$

One readily has that $\mathfrak{s}_{q}(\delta)=0$ if $d_{q}=1$ and otherwise $\mathfrak{s}_{q}(\delta)$ is a simple Lie subalgebra of $\mathfrak{g}$ where in fact

$$
\mathfrak{s}_{q}(\delta) \cong \operatorname{Lie} S l\left(d_{q}, \mathbb{C}\right)
$$

Let $\mathfrak{s}(\delta)$ be the semisimple Lie subalgebra given by putting

$$
\mathfrak{s}(\delta)=\oplus_{q=1}^{k} \mathfrak{s}_{q}(\delta) .
$$

Now let $\mathfrak{h}$ be the space of all diagonal matrices in $\mathfrak{g}$ so that $\mathfrak{h}$ is a Cartan subalgebra of $\mathfrak{g}$. Let

$$
\mathfrak{t}(\delta)=\left\{x \in \mathfrak{h} \mid a_{i i}(x)=a_{j j}(x) \text { if } q(i)=q(j)\right\}
$$

Let

$$
\mathfrak{m}(\delta)=\mathfrak{t}(\delta)+\mathfrak{s}(\delta)
$$


and put $\mathfrak{q}(\delta)=\mathfrak{m}(\delta)+\mathfrak{n}(\delta)$. The following proposition is straightforward and is left as an exercise.

Proposition 4.1. $\mathfrak{q}=\mathfrak{q}(\delta)$ is a parabolic subalgebra of $\mathfrak{g}$ and, up to conjugacy, every proper parabolic subalgebra is of this form. Moreover $\mathfrak{q}=\mathfrak{m}+\mathfrak{n}$ is a Levi decomposition of $\mathfrak{q}$ with $\mathfrak{n}$ as a nilradical and $\mathfrak{m}$ as a Levi factor where $\mathfrak{m}=\mathfrak{m}(\delta)$ and $\mathfrak{n}=\mathfrak{n}(\delta)$. Furthermore (4.10) is the decomposition (1.4) where $\mathfrak{t}=\mathfrak{t}(\delta)$ and $\mathfrak{s}=\mathfrak{s}(\delta)$. Next the set, $R$, of $\mathfrak{t}$-roots $\nu$ is parameterized by all pairs $r, s \in\{1, \ldots, k\}$, where $r \neq s$, and if the parameterization is denoted by $\nu(r, s)$, then for any $x \in \mathfrak{t}$ one has

$$
\nu(r, s)(x)=a_{i i}(x)-a_{j j}(x)
$$

for $i \in I_{r}$ and $j \in I_{s}$. In addition the $\mathfrak{t}$-root space corresponding to $\nu(r, s)$ is given by

$$
\mathfrak{g}_{\nu(r, s)}=\mathfrak{g}_{r, s}(\delta) .
$$

The irreducible adjoint action of $\mathfrak{m}$ on $\mathfrak{g}_{\nu(r, s)}$ is given as follows: Put $\mathfrak{s}_{q}=\mathfrak{s}_{q}(\delta)$. Then $\mathfrak{s}_{p}$ operates trivially if $p \notin\{r, s\}$. Furthermore $\mathfrak{g}_{\nu(r, s)}$ is one-dimensional if and only if $d_{r}=d_{s}=1$. If $d_{r}=1$ and $d_{s}>1$ (resp. $d_{r}>1$ and $d_{s}=1$ ), then $\mathfrak{g}_{\nu(r, s)}$ is $d_{s}$ (resp. $\left.d_{r}\right)$-dimensional and affords a fundamental irreducible $d_{s}$ (resp. $d_{r}$ )-dimensional of $\mathfrak{s}_{s}\left(\right.$ resp. $\left.\mathfrak{s}_{r}\right)$. Moreover if $d_{r}$ and $d_{s}$ are both greater than 1 , then $\operatorname{dim} \mathfrak{g}_{\nu(r, s)}=$ $d_{r} d_{s}$ and $\mathfrak{g}_{\nu(r, s)}$ affords the direct product of a fundamental irreducible $d_{r}$-dimensional representation of $\mathfrak{s}_{r}$ and a fundamental irreducible $d_{s}$-dimensional representation of $\mathfrak{s}_{s}$.

\section{References}

[B-de S] A. Borel and J. de Siebenthal, Les sous-groupes fermés de rang maximum des Lie clos. Comment. Math. Helv., 23 (1949), 200-221.

[Hum] J. Humphreys, Introduction to Lie Algebras and Representation Theory, Grad. Texts in Math., Vol. 9, Springer-Verlag, New York, 1972.

[Jac] N. Jacobson, Lie Algebras, Wiley(Interscience), New York, 10, 1962.

[Jos] A. Joseph, Orbital Varieties of the Minimal orbit, Ann. Ec. Norm. Sup.,31(1998), $17-45$.

[Kos] B. Kostant, A Characterization of the Classical Groups, Duke Math. J. 25:1(1958), 107-124. 
[Wol] J. Wolf, Spaces of Constant Curvature, McGraw-Hill, New York, 1967.

Bertram Kostant

Dept. of Math.

MIT

Cambridge, MA 02139

E-mail kostant@math.mit.edu 